\title{
Expressions and Clinical Significances of Angiopoietin-1, Angiopoietin-2, and Tie-2 Receptor in Patients With Colorectal Cancer
}

\author{
Sunghoon Hong, Hae Il Jung, Tae Sung Ahn, Han-Jo Kim ${ }^{1}$, Kyu-Taek Lee ${ }^{1}$, Moo-Jun Baek, Sang-Byung Bae ${ }^{1}$ \\ Department of Surgery, Soonchunhyang University Cheonan Hospital, Cheonan; ${ }^{1}$ Division of Hematology and Oncology, Department of \\ Internal Medicine, Soonchunhyang University Cheonan Hospital, Cheonan, Korea
}

Purpose: Angiopoietin-1 (Ang-1) plays a crucial role in vascular and hematopoietic development, mainly through its cognate receptor, Tie-2. Increased levels of Ang-2 have been shown to be correlated with abnormal tumor angiogenesis in several malignancies. Hence, we estimated the increased expression of Ang-2 relative to Ang- 1 in patients with colorectal cancer and correlated our finding with prognosis in order to investigate the relationships between the expressions of Ang-1/Ang-2/Tie-2 receptor and the clinical parameters or overall survival of such patients.

Methods: We retrospectively analyzed 114 tissue samples from patients with colorectal cancer by using immunohistochemistry (IHC) to examine Ang-1, Ang-2, and Tie-2 expressions and to investigate the relationship between those expressions and clinical parameters or overall survival of such patients. A Western blot analysis was used for Ang-2 expression.

Results: IHC staining showed a link between Ang-1 and Tie-2 ( $\mathrm{P}=0.018)$, as well as meaningful correlations between Ang-2 and Tie-2 receptor $(\mathrm{P}=0.022)$ and between lymph-node metastasis and Ang-2 (P = 0.025). The stronger the IHC staining for Ang-2 expression was, the shorter the cumulative survival was $(\mathrm{P}=0.016)$.

Conclusion: A relationship was found to exist between Ang-2 and Tie-2 expressions. The Ang-2 was correlated with lymph-node metastasis, and high expression of Ang-2 was indicative of poor overall survival. These findings suggest that Ang-2 is a useful prognostic marker in the management of patients with colorectal cancer. In addition, we suggest that Ang/Tie-2 signaling plays an important role in the progression of colorectal cancer.

Keywords: Colorectal neoplasms; Angiopoietins; TIE-2 receptor; Prognosis

\section{INTRODUCTION}

The formation of new blood and lymphatic vessels from preexisting tumors is considered an essential step in tumor progression and distant metastasis; therefore, the use of antiangiogenic drugs is an important clinical strategy [1]. Angiogenesis indicates the

Received: October 14, 2016 - Accepted: October 24, 2016

Correspondence to: Sang-Byung Bae, M.D.

Division of Hematology and Oncology, Department of Internal Medicine, Soonchunhyang University Cheonan Hospital, 31 Suncheonhyang 6-gil, Dongnam-gu, Cheonan 31151, Korea

Tel: +82-41-570-3667, Fax: +82-41-574-5762

E-mail: baesan@schmc.ac.kr

(C) 2017 The Korean Society of Coloproctology

This is an open-access article distributed under the terms of the Creative Commons Attribution NonCommercial License (http://creativecommons.org/licenses/by-nc/4.0) which permits unrestricted noncommercial use, distribution, and reproduction in any medium, provided the original work is properly cited. development of new blood vessels from the preexisting vasculature. It is a normal physiological process essential for the growth and development of normal tissues [2]; however, it is also recognized as an initial step in tumor progression [3]. The first observation that the growth of human tumors is characterized by an increase in vessel density was made approximately 100 years ago. In the late 1930s, the existence of tumor-derived factors able to promote new vessel formation was first considered [4]. The moststudied signaling pathway for angiogenesis, and the one to which most of the efforts of the pharmaceutical industry have been devoted, is that of the vascular endothelial growth factor (VEGF) and its receptors, mainly VEGFR-2. However, angiopoietin-1 (Ang-1) and Ang-2 and their receptor Tie-2 represent another major tyrosine kinase-ligand system that is fundamental for the regulation of angiogenesis $[1,5,6]$.

Ang-2 is part of the Ang/Tie signaling pathway, which is in- 
volved in vascular growth and maturation. In the presence of a Tie-2 agonist, Ang-1 and Ang-2 may act as antagonists, blocking Tie-2 signaling, although their actions are largely context-dependent $[3,5]$. Ang-1/Tie-2 signaling confers structural stability to mature vessels and protects the endothelium from activation by exogenous cytokines, including angiogenetic and inflammatory stimuli, whereas Ang-2 prevents Ang-1 from stimulating vessel remodeling, causing a loss of the pericyte wall and endothelial cell (EC) junctions [7]. Ang-2/Tie-2 signaling promotes the destabilization of quiescent ECs and enables VEGF-induced angiogenesis $[6,7]$. In this context, Ang-2 induced effects on the existing vasculature lead, in the presence of pro-angiogenic factors (including VEGF, fibroblast [FGF], and platelet-derived growth factor [PDGF]), to the formation of novel vessels, suggesting that the ratio of Ang-1 to Ang-2 plays an important role during physiological angiogenesis [8]. However, increased levels of Ang-2 have been correlated with abnormal tumor angiogenesis in several malignancies, including hepatocelluar carcinomas, metastatic colorectal cancer, breast cancer, non-small-cell lung cancer, prostate cancer, and ovarian cancer [9]. For these reasons, we estimated the increased expression of Ang- 2 relative to that of Ang-1 in patients with colorectal cancer and examined the correlation of that ratio with a poor prognosis. The purposes of this study were to investigate the expressions of Ang-1, Ang-2, and Tie-2 at different stages of colorectal cancer and to elucidate the relationships between the clinical outcome and the expressions of Ang-1, Ang2 , and Tie- 2 in patients with colorectal cancer.

\section{METHODS}

\section{Patients and specimens}

This study included patients with a pathologically and surgeryconfirmed colorectal adenocarcinoma. All patients were identified from a case-control study of patients with colorectal cancer conducted at Soonchunhyang Cheonan Hospital from February of 2002 to December of 2007. Each cancer was assigned a histological type according to the World Health Organization classification [10]. Tumor stage was divided according to the TNM staging system published by the American Joint Committee on Cancer.

\section{IHC staining}

Patients' tissue specimens, obtained during surgery, were examined by IHC staining using a tissue microarray method to assess Ang-1, Ang-2, and Tie-2 expressions. The results were categorized from negative to tumor +1 and tumor +2 according to the strength of the staining for Ang-1, Ang-2, and Tie-2 (Fig. 1). Moreover, Ang-2 expression in the colorectal carcinoma tissues was compared to that in normal tissues by using Western blot analyses.

\section{Western blot assay}

Cancer cells were washed with phosphate-buffered saline and lysed using Pro-Prep (iNtRON, Seongnam, Korea) at $4^{\circ} \mathrm{C}$ overnight. The supernatant was collected after centrifugation. Protein concentrations were determined using a Pierce Bicinchoninic Acid Protein Assay Kit (Thermo Fisher Scientific, Rockfold, IL, USA). Cell lysate samples $(30 \mu \mathrm{g})$ were subjected to $10 \%$ sodium dodecyl sulfate-polyacrylamide gel electrophoresis (110 V for 2 hours) and then transferred to a polyvinylidene fluoride membrane by using the Trans-Blot Turbo system (Bio-Rad Laboratories, Hercules, CA, USA). The membrane was blocked for 1 hour in blocking buffer ( $5 \%$ nonfat skim milk/1X TBS-T [Tris-buffered saline with $0.1 \%$ Tween 20]) at room temperature, followed by incubation with primary antibodies at $4^{\circ} \mathrm{C}$ overnight, including monoclonal antibodies against Ang-2 diluted in 1X TBS-T (1:1,000; R\&D System Inc., Minneapolis, MN, USA). After the membrane had been washed five times with TBS-T, it was incubated with goat anti-mouse (1:80,000 dilution in 1X TBS-T; Sigma-Aldrich, St. Louis, MO, USA) secondary antibodies for 1 hour at room temperature. The membrane was then washed 4 or 5 times in $1 \mathrm{X}$ TBS-T for 1 hour. The signal was detected with an enhanced chemiluminescence kit by using ECM solution (Advansta, Menlo Park, CA, USA) and a Molecular Imager ChemiDoc XRS + (Bio-Rad Laboratories). $\beta$-Actin was used as a loading control.

\section{Statistical analyses}

Overall survival (OS) was calculated from the date of diagnosis to the date of death. The data were analyzed statistically using SPSS ver. 14.0 (SPSS Inc., Chicago, IL, USA). A Kaplan-Meier plot was used to estimate OS curves. The survival curves of the 2 groups were compared using the log-rank test. Chi-square analyses were used to compare the association of Ang-1, Ang-2, or Tie-2 expression with other clinical variables. Two-sided $95 \%$ confidence intervals were estimated. $\mathrm{P}<0.05$ was taken as statistically significant.

\section{RESULTS}

In total, 114 males and females were included in this study. The mean age of the patients at the time of diagnosis was 63.7 years (range, 33-85 years). The majority of the patients were male (61.4\%). Most of the patients had TNM stage II or III colorectal cancer $(76.3 \%)$. The mean follow-up time was 5.5 years. Of the 114 patients, 31 (27.2\%), 76 (66.6\%), and 68 (59.6\%) showed positive immunohistochemistry (IHC) staining for Ang-1, Ang-2, and Tie-2, respectively.

Generally, in comparison with normal tissue, Western blot analyses showed higher expression of Ang-2 in colorectal cancer tissue (Fig. 2). Consequently, in 8 out of the 10 colorectal-cancer tissue samples, the expression of Ang-2 was higher than it was in normal tissue samples. In terms of correlations between the Ang1, Ang-2, and Tie-2 proteins, no statistically significant relationship was found between the Ang-1 and the Ang-2 expressions ( $P$ 


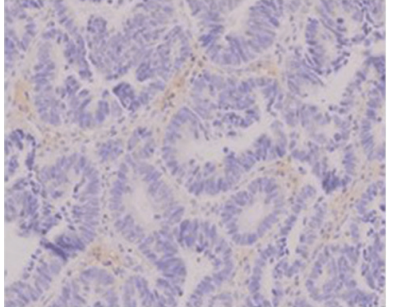

Normal

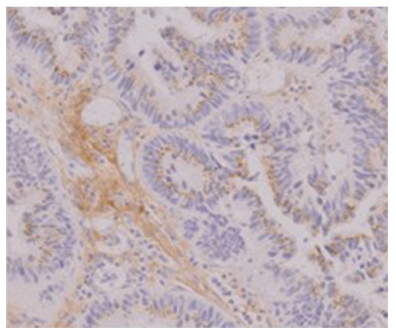

Tumor $(+)$

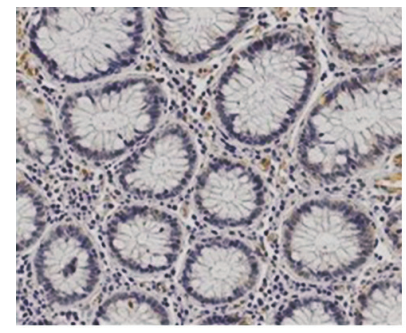

Normal

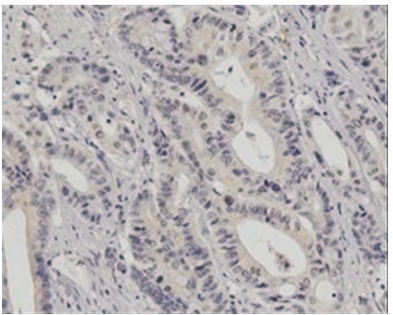

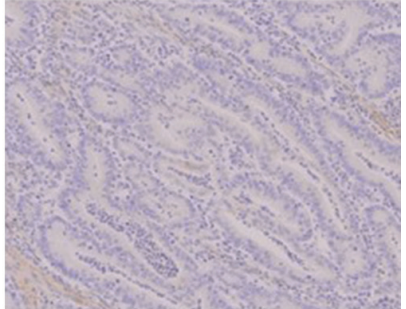

Tumor (-)

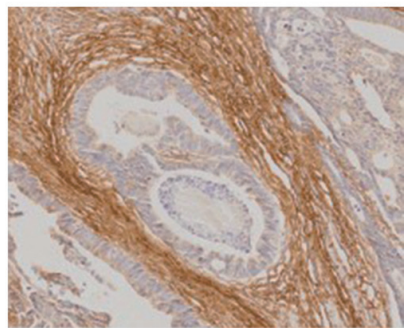

Tumor $(++)$

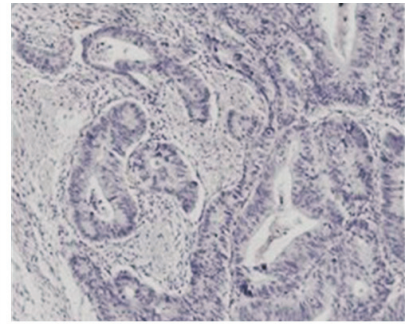

Tumor (-)

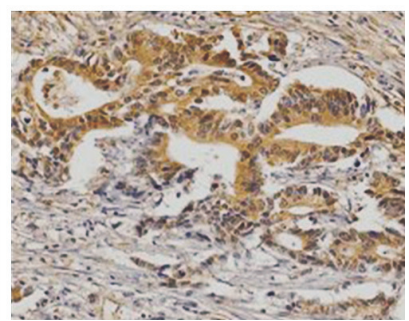

Tumor (+)

Tumor $(++)$

C

Ang-2 (62-70 kDa)

$\begin{array}{llllllllll}\mathrm{N} & \mathrm{T} & \mathrm{N} & \mathrm{T} & \mathrm{N} & \mathrm{T} & \mathrm{N} & \mathrm{T} & \mathrm{N} & \mathrm{T}\end{array}$

$\beta$-Actin (42 kDa)

Ang-2 (62-70 kDa)

$\beta$-Actin (42 kDa)

Fig. 2. Ang-2 expression in colorectal cancer tissues. Ang-2 expression was higher in 8 out of 10 tumor tissue samples compared with normal tissue. $\mathrm{N}$, normal tissue; $\mathrm{T}$, tumor.

$=0.137$ ) (Table 1A), but a meaningful statistically significant relationship was found between the Ang-1 and the Tie-2 expressions

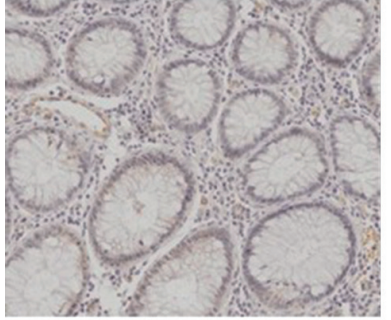

Normal

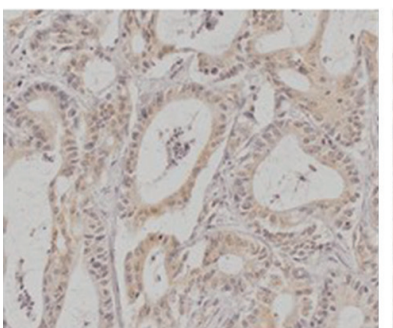

Tumor (+)

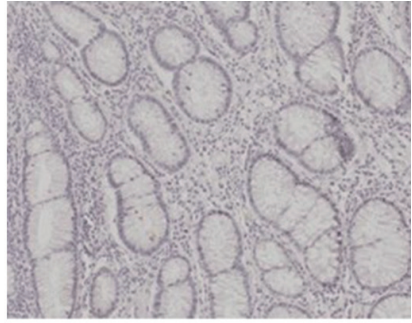

Tumor (-)

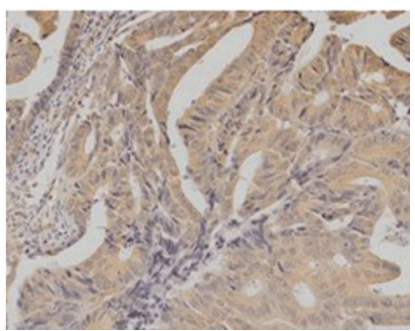

Tumor (++)
B
Fig. 1. Overexpressions of angiopoietin-1 (Ang-1), angiopoietin-2, and their receptor Tie-2: (A) Ang-1 overexpression: normal status to tumor 3 positive status based on the strength of immunohistochemistry (IHC) staining, (B) Ang-2 overexpression: normal status to tumor 3 positive status based on the strength of IHC staining. (C) Tie-2 overexpression: normal status to tumor 3 positive status based on the strength of IHC staining $(\mathrm{H} \& \mathrm{E}, \times 200)$.

$(\mathrm{P}=0.018)$ (Table 1B). Furthermore, no strong correlation was found between the Ang-2 and the Tie-2 expressions $(\mathrm{P}=0.022)$ (Table 1C).

The patients' clinical factors (e.g., age, sex, tumor location, differentiation, preoperative carcinoembryonic antigen [CEA] level, T stage, $\mathrm{N}$ stage, lymphatic invasion, venous invasion and pathologic stage) showed no correlations to Ang-1 expression (Table 2). With respect to Ang-2, all correlations were negative except for lymph-node metastasis $(\mathrm{P}=0.025)$ (Table 3$)$. The Tie-2 expression was correlated with age and preoperative CEA level ( $\mathrm{P}=$ $0.046, \mathrm{P}=0.044$, respectively) (Table 4 ). In analyses of the survival data, Ang-1, Ang-2, and Tie-2 expressions showed no statistical relationships with cumulative survival. However, subpopulation analyses based on low expression (negative, +1) and high expres- 
Table 1. Relationships between the expressions of angiopoietins; Ang-1 and Ang-2 (A), Ang-1 and Tie-2 (B), Tie-2 and Ang-2 (C) (A)

\begin{tabular}{lrrrc}
\hline & \multicolumn{4}{c}{ Ang-2 expression } \\
\cline { 2 - 5 } & Cases & Negative & Positive & P-value \\
\hline Ang-1 expression & & & & 0.137 \\
$\quad$ Negative & $83(100)$ & $31(37.3)$ & $52(62.7)$ & \\
Positive & $31(100)$ & $7(22.6)$ & $24(77.4)$ & \\
\hline
\end{tabular}

(B)

\begin{tabular}{lrrrr}
\hline & \multicolumn{4}{c}{ Tie-2 expression } \\
\cline { 2 - 5 } & Cases & Negative & Positive & P-value \\
\hline Ang-1 expression & & & & $0.018^{*}$ \\
$\quad$ Negative & $83(100)$ & $39(47.0)$ & $44(53.0)$ & \\
Positive & $31(100)$ & $7(22.6)$ & $24(77.4)$ \\
\hline
\end{tabular}

(C)

\begin{tabular}{lcccc}
\hline & \multicolumn{4}{c}{ Ang-2 expression } \\
\cline { 2 - 5 } & Cases & Negative & Positive & P-value \\
\hline Tie-2 expression & & & & $0.022^{\dagger}$ \\
Negative & $46(100)$ & $21(45.7)$ & $25(54.3)$ \\
Positive & $68(100)$ & $17(25.0)$ & $51(75.0)$ \\
\hline
\end{tabular}

Values are presented as number (\%).

No relationship was found between Ang-1 and Ang-2; however, statistically meaningful relationships were found between Ang- 1 and Tie-2 ( $\left.{ }^{*} P<0.05\right)$ and between Ang-2 and Tie-2 ( $\left.{ }^{+} \mathrm{P}<0.05\right)$.

sion $(+2)$ showed that high expression of Ang-2 had an important statistically significant relationship with cumulative survival $(\mathrm{P}=$ 0.016). The stronger the IHC staining of Ang-2 was, the shorter the cumulative survival was (Fig. 3).

\section{DISCUSSION}

Angiogenesis is a normal and vital process in growth and development, as well as in wound healing and in granulation tissue, including EC migration toward stimuli in the microenvironment, EC proliferation, tube formation, and intussusception [11]. Also, the growth and progression of human tumors is well known to depend on the pathologic growth of new blood vessels. Among the several regulators of angiogenesis, the most relevant signaling pathways for angiogenesis are the VEGF-related pathway and its receptors (VEGFR-1, -2, and -3). The approval of bevacizumab, an anti-VEGF monoclonal antibody, for the treatment of several cancers was based on successful clinical trials and a firm biological rationale [8]. However, the development of resistance to VEGF inhibitors has begun to emerge as a major issue. Indeed, tumors are now known to be able to circumvent VEGF inhibition through multiple mechanisms, including changes in both tumor cells and tumor-associated ECs. Recent studies have also demonstrated that a prolonged VEGFR blockade, after initial stabilization of the
Table 2. Relationships between Ang-1 expression and clinical factors

\begin{tabular}{|c|c|c|c|c|}
\hline \multirow[b]{2}{*}{ Variable } & \multicolumn{3}{|c|}{ Ang-1 } & \multirow[b]{2}{*}{ P-value } \\
\hline & $\begin{array}{c}(-) \\
(n=83)\end{array}$ & $\begin{array}{c}1+ \\
(n=26)\end{array}$ & $\begin{array}{c}2+ \\
(n=5)\end{array}$ & \\
\hline Age (yr) & & & & 0.750 \\
\hline$<60$ & 27 (32.5) & $7(26.9)$ & $1(20.0)$ & \\
\hline$\geq 60$ & $56(67.5)$ & 19 (73.1) & $4(80.0)$ & \\
\hline Sex, male : female & $54: 29$ & $12: 14$ & $4: 1$ & 0.153 \\
\hline Tumor location & & & & 0.587 \\
\hline Caecum, ascending, transverse & $18(21.7)$ & $5(19.2)$ & $2(40.0)$ & \\
\hline Descending, sigmoid, rectum & 65 (78.3) & $21(80.8)$ & $3(60.0)$ & \\
\hline Differentiation & & & & 0.506 \\
\hline Well, moderate & $75(90.4)$ & 25 (96.2) & $5(100)$ & \\
\hline Poor, mucinous & $8(9.6)$ & $1(3.8)$ & $0(0)$ & \\
\hline Preoperative CEA (ng/mL) & & & & 0.638 \\
\hline$<5$ & $52(62.7)$ & $18(69.2)$ & $4(80.0)$ & \\
\hline$\geq 5$ & $31(37.3)$ & $8(30.8)$ & $1(20.0)$ & \\
\hline T stage & & & & 0.284 \\
\hline pT1 & $3(3.6)$ & $1(3.8)$ & $1(20.0)$ & \\
\hline pT2 & $18(21.7)$ & $4(15.4)$ & $0(0)$ & \\
\hline pT3 & $51(61.4)$ & $20(76.9)$ & $4(80.0)$ & \\
\hline pT4 & $11(13.3)$ & $1(3.8)$ & $0(0)$ & \\
\hline N stage & & & & 0.111 \\
\hline Negative & $48(57.8)$ & $9(34.6)$ & $3(60.0)$ & \\
\hline Positive & 35 (42.2) & $17(65.4)$ & $2(40.0)$ & \\
\hline Lymphatic invasion & & & & 0.450 \\
\hline Negative & $66(72.5)$ & $22(24.2)$ & $3(3.3)$ & \\
\hline Positive & $17(73.9)$ & $4(17.4)$ & $2(8.7)$ & \\
\hline Venous invasion & & & & 0.941 \\
\hline Negative & 68 (81.9) & 22 (84.6) & $4(80.0)$ & \\
\hline Positive & 15 (18.1) & $4(15.4)$ & $1(20.0)$ & \\
\hline Pathologic stage & & & & 0.456 \\
\hline I & 17 (20.5) & $2(7.7)$ & $1(20.0)$ & \\
\hline$\|$ & $29(34.9)$ & $7(26.9)$ & $2(40.0)$ & \\
\hline III & $31(37.3)$ & $16(61.5)$ & $2(40.0)$ & \\
\hline IV & $6(7.2)$ & $1(3.8)$ & $0(0)$ & \\
\hline
\end{tabular}

Values are presented as number (\%).

CEA, carcinoembryonic antigen.

disease, is followed by the up-regulation of other pro-angiogenic factors, FGF, PDGF, ephrins, and angiopoietins [12]. At present, moving beyond VEGF, Ang-1, Ang-2 and their receptor, Tie-2, are recognized as another major tyrosine kinase-ligand system involved in the regulation of angiogenesis $[5,6]$.

Ang-1 and Ang-2 interact with Tie-2, which is most frequently 
Table 3. Relationships between Ang-2 expression and clinical factors

\begin{tabular}{|c|c|c|c|c|}
\hline \multirow[b]{2}{*}{ Variable } & \multicolumn{3}{|c|}{ Ang-2 } & \multirow[b]{2}{*}{ P-value } \\
\hline & $\begin{array}{c}(-) \\
(n=38)\end{array}$ & $\begin{array}{c}1+ \\
(n=47)\end{array}$ & $\begin{array}{c}2+ \\
(n=29)\end{array}$ & \\
\hline Age (yr) & & & & 0.831 \\
\hline$<60$ & $13(34.2)$ & $14(29.8)$ & $8(27.6)$ & \\
\hline$\geq 60$ & $25(65.8)$ & $33(70.2)$ & $21(72.4)$ & \\
\hline Sex, male : female & $23: 15$ & $30: 17$ & $17: 12$ & 0.894 \\
\hline Tumor location & & & & 0.950 \\
\hline Caecum, ascending, transverse & $8(21.1)$ & $11(23.4)$ & $6(20.7)$ & \\
\hline Descending, sigmoid, rectum & $30(78.9)$ & $36(76.6)$ & $23(79.3)$ & \\
\hline Differentiation & & & & 0.304 \\
\hline Well, moderate & $33(86.8)$ & $44(93.6)$ & $28(96.6)$ & \\
\hline Poor, mucinous & $5(13.2)$ & $3(6.4)$ & $1(3.4)$ & \\
\hline Preoperative CEA (ng/mL) & & & & 0.696 \\
\hline$<5$ & $25(65.8)$ & $32(68.1)$ & $17(58.6)$ & \\
\hline$\geq 5$ & $13(34.2)$ & $15(31.9)$ & $12(41.4)$ & \\
\hline T stage & & & & 0.698 \\
\hline pT1 & $3(7.9)$ & $2(4.3)$ & $0(0)$ & \\
\hline pT2 & $6(15.8)$ & $8(17.0)$ & $8(27.6)$ & \\
\hline pT3 & $25(65.8)$ & $32(68.1)$ & $18(62.1)$ & \\
\hline pT4 & $4(10.5)$ & $5(10.6)$ & $3(10.3)$ & \\
\hline $\mathrm{N}$ stage & & & & 0.025 \\
\hline Negative & $22(57.9)$ & $29(61.7)$ & $9(31.0)$ & \\
\hline Positive & $16(42.1)$ & 18 (38.3) & $20(69.0)$ & \\
\hline Lymphatic invasion & & & & 0.250 \\
\hline Negative & 28 (73.7) & 41 (87.2) & 22 (75.9) & \\
\hline Positive & $10(26.3)$ & $6(12.8)$ & $7(24.1)$ & \\
\hline Venous invasion & & & & 0.254 \\
\hline Negative & $29(76.3)$ & 42 (89.4) & $23(79.3)$ & \\
\hline Positive & $9(23.7)$ & $5(10.6)$ & $6(20.7)$ & \\
\hline Pathologic stage & & & & 0.219 \\
\hline I & $8(21.1)$ & $8(17.0)$ & $4(13.8)$ & \\
\hline$\|$ & $14(36.8)$ & $19(40.4)$ & $5(17.2)$ & \\
\hline III & $15(39.5)$ & $16(34.0)$ & $18(62.1)$ & \\
\hline IV & $1(2.6)$ & $4(8.5)$ & $2(6.9)$ & \\
\hline
\end{tabular}

Values are presented as number (\%).

CEA, carcinoembryonic antigen.

expressed in ECs. Upon binding an Ang, Tie-2 dimerizes, and its tyrosine residues are phosphorylated. In the blood vascular endothelium, Ang-1 exerts agonistic functions via increased phosphorylation of Tie-2 [13]. Both Ang-1 and Tie-2 are essential for the remodeling of a functional blood vessel network during embryogenesis. They also promote various functions characteristic
Table 4. Relationships between Tie-2 expression and clinical factors

\begin{tabular}{|c|c|c|c|c|}
\hline \multirow[b]{2}{*}{ Variable } & \multicolumn{3}{|c|}{ Tie-2 } & \multirow[b]{2}{*}{ P-value } \\
\hline & $\begin{array}{c}(-) \\
(n=46)\end{array}$ & $\begin{array}{c}1+ \\
(n=36)\end{array}$ & $\begin{array}{c}2+ \\
(n=32)\end{array}$ & \\
\hline Age (yr) & & & & 0.046 \\
\hline$<60$ & $20(43.5)$ & $7(19.4)$ & $8(25.0)$ & \\
\hline$\geq 60$ & $26(56.5)$ & $29(80.6)$ & $24(75.0)$ & \\
\hline Sex, male : female & $29: 17$ & $20: 16$ & $21: 11$ & 0.666 \\
\hline \multicolumn{5}{|l|}{ Tumor location } \\
\hline Caecum, ascending, transverse & $10(21.7)$ & $10(27.8)$ & $5(15.6)$ & 0.481 \\
\hline Descending, sigmoid, rectum & $36(78.3)$ & $26(72.2)$ & 27 (84.4) & \\
\hline Differentiation & & & & 0.815 \\
\hline Well, moderate & $42(91.3)$ & $34(94.4)$ & 29 (90.6) & \\
\hline Poor, mucinous & $4(8.7)$ & $2(5.6)$ & $3(9.4)$ & \\
\hline Preoperative CEA (ng/mL) & & & & 0.044 \\
\hline$<5$ & $36(78.3)$ & $21(58.3)$ & $17(53.1)$ & \\
\hline$\geq 5$ & $10(21.7)$ & $15(41.7)$ & $15(46.9)$ & \\
\hline T stage & & & & 0.582 \\
\hline pT1 & $3(6.5)$ & $1(2.8)$ & $1(3.1)$ & \\
\hline pT2 & $12(26.1)$ & $5(13.9)$ & $5(15.6)$ & \\
\hline pT3 & $26(56.5)$ & $25(69.4)$ & $24(75.0)$ & \\
\hline pT4 & $5(10.9)$ & $5(13.9)$ & $2(6.3)$ & \\
\hline N stage & & & & 0.085 \\
\hline Negative & $29(63.0)$ & 19 (52.8) & $12(37.5)$ & \\
\hline Positive & $17(37.0)$ & 17 (47.2) & $20(62.5)$ & \\
\hline Lymphatic invasion & & & & 0.119 \\
\hline Negative & 41 (89.1) & $27(75.0)$ & 23 (71.9) & \\
\hline Positive & $5(10.9)$ & $9(25.0)$ & $9(28.1)$ & \\
\hline Venous invasion & & & & 0.243 \\
\hline Negative & 41 (89.1) & $27(75.0)$ & 26 (81.3) & \\
\hline Positive & $5(10.9)$ & $9(25.0)$ & $6(18.8)$ & \\
\hline Pathologic stage & & & & 0.105 \\
\hline I & $12(26.1)$ & $3(8.3)$ & $5(15.6)$ & \\
\hline$\|$ & $16(34.8)$ & $16(44.4)$ & $6(18.8)$ & \\
\hline III & 16 (34.8) & $14(38.9)$ & 19 (59.4) & \\
\hline IV & $2(4.3)$ & $3(8.3)$ & $2(6.3)$ & \\
\hline
\end{tabular}

Values are presented as number (\%).

CEA, carcinoembryonic antigen.

of the mature blood vasculature, including EC survival [14]. Ang-2 is part of the Ang/Tie-2 signaling pathway, which is involved in vascular growth and maturation [5]. Ang-2 expression is increased in activated and hypoxic vascular ECs in tumors, leading to increased cell survival. Ang-2 induces vascular destabilization during tumor metastasis, suggesting that during a VEGF 

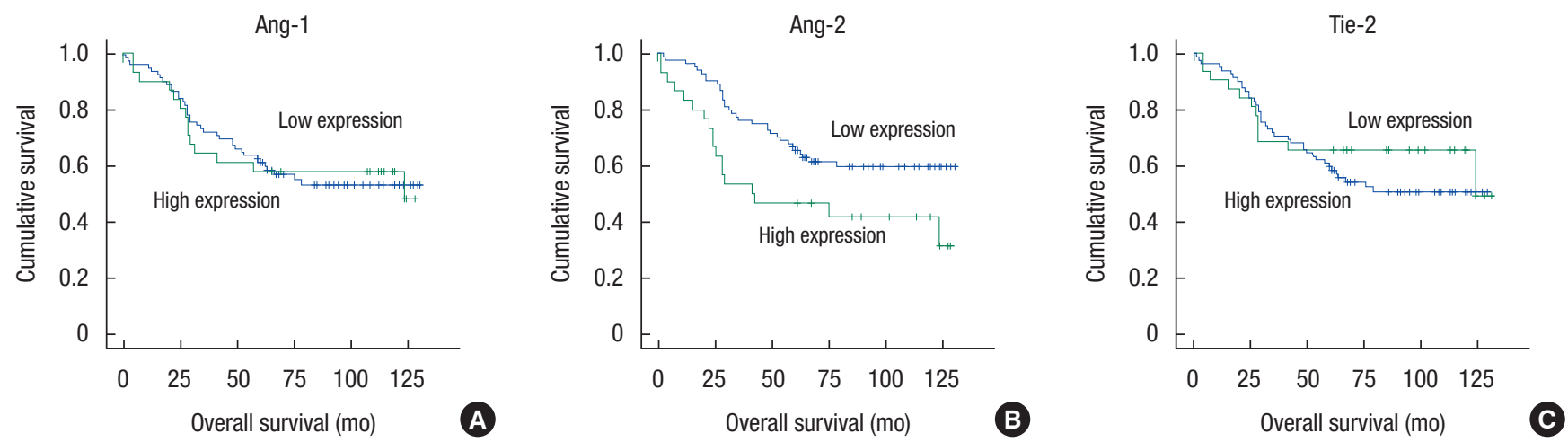

Fig. 3. Relationship between Tie-2 and overall survival. (A) Subpopulation analysis of Ang-1 expression based on low expression (negative, +1 ) and high expression (+2). High expression of Ang-1 had no statistical significance $(\mathrm{P}=0.941)$. (B) Subpopulation analysis of Ang-2 expression based on low expression (negative, +1$)$ and high expression $(+2)$. High expression of Ang-2 had important statistical significance $(\mathrm{P}$ $=0.016$ ). (C) Subpopulation analysis of Tie-2 expression based on low expression (negative, +1 ) and high expression $(+2)$. High expression of Tie-2 had no statistical significance $(\mathrm{P}=0.476)$.

blockade, Ang-2 induced vascular destabilization is associated with increased metastasis [8]. In several human cancers, an intimate connection has been shown between an increased Ang-2 level and tumor progression. Ang-2 plays an important role in cancer progression (growth, invasion, and metastasis) and may represent a poor clinical outcome [15]. Systemic overexpression of Ang-2 promotes metastatic dissemination [16].

On the basis of the above knowledge, we presumed that most cancer cells wound utilize the Ang/Tie-2 signaling pathway. We suggested that Ang-1, Ang-2, and Tie-2 overexpressions could be detected using IHC staining in colorectal cancer tissues (Fig. 1), with a significant correlations for Ang-2/Tie-2 (Table 1B) and Tie-2/Ang-1 (Table 1C). Moreover, compared to several clinical factors, Ang-2 only had a meaningful relationship with lymphnode metastasis and the patient's clinical outcome (Table 3). Holopainen et al. [16] showed previously that Ang-2 affected tumor lymphangiogenesis and metastasis, especially in patients with lung cancer. Similarly, our results showed that systemic Ang-2 overexpression promoted lymph-node metastasis due to tumor growth and angiogenesis, as well as poor OS. Until now, no definite meaningful prognostic markers for patients with colorectal cancer have been identified. However, our results indicate that high expression of Ang-2 may be a useful prognostic marker in the management of patients with a colorectal carcinoma. Our results also suggest a possible role for the Ang/Tie-2 system in the progression of colorectal cancer.

Our results suggest that colorectal cancer involves Ang- 1 and Ang-2 overexpressions and that an especially-high expression of Ang-2 is a meaningful overall predictor of survival, sadly leading to a poor prognosis. This indicates that high expression of Ang-2 may be a useful prognostic marker in the management of patients with colorectal cancer. Also, we suggest that the Ang/Tie-2 signaling pathway plays an important role in the progression of colorectal cancer.

\section{CONFLICT OF INTEREST}

No potential conflict of interest relevant to this article was reported.

\section{ACKNOWLEDGMENTS}

This work was supported by the Soonchunhyang University Research Fund.

\section{REFERENCES}

1. Albini A, Noonan DM. Angiopoietin2 and tie2: tied to lymphangiogenesis and lung metastasis. New perspectives in antimetastatic antiangiogenic therapy. J Natl Cancer Inst 2012;104:429-31.

2. Petrillo M, Scambia G, Ferrandina G. Novel targets for VEGF-independent anti-angiogenic drugs. Expert Opin Investig Drugs 2012;21:451-72.

3. Hanahan D, Weinberg RA. Hallmarks of cancer: the next generation. Cell 2011;144:646-74.

4. Ferrara N. VEGF and the quest for tumour angiogenesis factors. Nat Rev Cancer 2002;2:795-803.

5. Saharinen P, Eklund L, Pulkki K, Bono P, Alitalo K. VEGF and angiopoietin signaling in tumor angiogenesis and metastasis. Trends Mol Med 2011;17:347-62.

6. Huang H, Bhat A, Woodnutt G, Lappe R. Targeting the ANGPTTIE2 pathway in malignancy. Nat Rev Cancer 2010;10:575-85.

7. Maisonpierre PC, Suri C, Jones PF, Bartunkova S, Wiegand SJ, Radziejewski C, et al. Angiopoietin-2, a natural antagonist for Tie2 that disrupts in vivo angiogenesis. Science 1997;277:55-60.

8. Sato TN, Tozawa Y, Deutsch U, Wolburg-Buchholz K, Fujiwara Y, Gendron-Maguire M, et al. Distinct roles of the receptor tyrosine kinases Tie-1 and Tie-2 in blood vessel formation. Nature 1995; 376:70-4. 
9. Ahmad SA, Liu W, Jung YD, Fan F, Wilson M, Reinmuth N, et al. The effects of angiopoietin-1 and -2 on tumor growth and angiogenesis in human colon cancer. Cancer Res 2001;61:1255-9.

10. Jass SR, Sobin LH. Histological typing of intestinal tumors. 2nd ed. World Health Organization. International Histological Classification of Tumours. Berlin: Springer-Verlg; 1989.

11. Carmeliet P, Jain RK. Angiogenesis in cancer and other diseases. Nature 2000;407:249-57.

12. Casanovas O, Hicklin DJ, Bergers G, Hanahan D. Drug resistance by evasion of antiangiogenic targeting of VEGF signaling in latestage pancreatic islet tumors. Cancer Cell 2005;8:299-309.

13. Eklund L, Olsen BR. Tie receptors and their angiopoietin ligands are context-dependent regulators of vascular remodeling. Exp
Cell Res 2006;312:630-41.

14. Kim I, Kim HG, So JN, Kim JH, Kwak HJ, Koh GY. Angiopoietin-1 regulates endothelial cell survival through the phosphatidylinositol 3'-Kinase/Akt signal transduction pathway. Circ Res 2000;86:24-9.

15. Lemieux C, Maliba R, Favier J, Théorêt JF, Merhi Y, Sirois MG. Angiopoietins can directly activate endothelial cells and neutrophils to promote proinflammatory responses. Blood 2005;105: 1523-30.

16. Holopainen T, Saharinen P, D’Amico G, Lampinen A, Eklund L, Sormunen R, et al. Effects of angiopoietin-2-blocking antibody on endothelial cell-cell junctions and lung metastasis. J Natl Cancer Inst 2012;104:461-75. 